\title{
Timing of administration of epinephrine predicts the responsiveness to epinephrine in norepinephrine-refractory septic shock: a retrospective study
}

\author{
Daisuke Kasugai ${ }^{{ }^{*}}$ D, Mitsuaki Nishikimi ${ }^{1}$, Kazuki Nishida², Michiko Higashi', Takanori Yamamoto', \\ Atsushi Numaguchi ${ }^{1}$, Kunihiko Takahashi ${ }^{2}$, Shigeyuki Matsui ${ }^{2}$ and Naoyuki Matsuda ${ }^{1}$
}

\begin{abstract}
Background: Currently, the appropriate method of management of patients with refractory septic shock remains unclear. This study aimed to evaluate the factors associated with response to epinephrine in norepinephrinerefractory septic shock.

Methods: A retrospective single-center observational study was performed using data from adult patients ( $\geq 18$ years old) admitted to our emergency and medical intensive care unit (ICU) from January 2014 to December 2017 who had received epinephrine to treat norepinephrine-refractory septic shock. The response was considered positive if there was increase in mean arterial pressure of $10 \mathrm{mmHg}$ or decrease in arterial lactate level $3 \mathrm{~h}$ after epinephrine administration.
\end{abstract}

Results: Forty-one patients were included: 24 responders (59\%) and 17 non-responders (41\%). Responders showed higher rate of survival from shock ( $92 \%$ vs. $18 \% ; P<0.001)$, and 28 -day survival $(83 \%$ vs. $18 \% ; P<0.001)$. In multivariable analysis, time of epinephrine administration after ICU admission (odds ratio [OR] 0.48; 95\% confidence interval $[\mathrm{Cl}] 0.27-0.87 ; P=0.011)$ and SOFA score (OR 0.19; $95 \% \mathrm{Cl} 0.04-0.88 ; P=0.034)$ were associated with epinephrine response. Time of epinephrine administration was also significantly associated with survival from shock (OR 0.42; $P=0.005)$ and 28-day survival (OR 0.14; $P=0.006)$, while SOFA score did not. Using inverse probability of treatment weighing (IPTW) adjustment of propensity score, epinephrine administration later than $24 \mathrm{~h}$ after ICU admission was associated with poor response (OR 0.07; 95\% Cl 0.02-0.21; $P<0.001$ ).

Conclusions: Early administration of epinephrine after ICU admission (i.e., within $24 \mathrm{~h}$ ) is associated with better hemodynamic status in patients with refractory septic shock.

Keywords: Refractory septic shock, Sepsis, Norepinephrine, Epinephrine

\section{Background}

Refractory shock is defined as resistance to the standard dose of norepinephrine administered as a first-line vasopressor [1] and is the major reason for death among patients with sepsis or septic shock [2]. Despite the high

\footnotetext{
* Correspondence: daisuke99999@icloud.com

This study was performed at Nagoya University Graduate School of Medicine, Nagoya, Japan.

${ }^{1}$ Department of Emergency and Critical Care, Nagoya, University Graduate School of Medicine, Tsurumai-cho 64, Syowa-ku, Nagoya, Aichi 4668560, Japan

Full list of author information is available at the end of the article
}

mortality rate in patients with refractory septic shock [3-5], the appropriate management remains unclear.

A couple of guidelines suggest treatment options, including the use of epinephrine, for patients with septic shock who are refractory to norepinephrine $[6,7]$. Nonetheless, the extent of the effectiveness of such treatments is unclear. Epinephrine was previously shown to have some beneficial effects against refractory septic shock in rats [8]. In clinical studies, Le Tulzo et al. also showed that epinephrine improves right ventricular contractility in septic shock patients who are unresponsive to dobutamine

(c) The Author(s). 2019 Open Access This article is distributed under the terms of the Creative Commons Attribution 4.0 International License (http://creativecommons.org/licenses/by/4.0/), which permits unrestricted use, distribution, and 
[9], while Mahmoud et al., in a randomized control trial, showed that compared to dobutamine, epinephrine effectively improved mean arterial pressure (MAP) and cardiac index in 60 septic shock patients resistant to norepinephrine [10]. Conversely, other large randomized controlled trials did not find epinephrine to be superior to other vasopressors or inotropic agents [11, 12].

One of the most important reasons that these randomized controlled trials failed to demonstrate the effectiveness of epinephrine may be the heterogeneity of patients with sepsis, as some are not responsive to epinephrine. If epinephrine responders can be identified in the earlier phases of septic shock, epinephrine could be used more effectively to treat such patients while alternative treatments can more promptly be considered for non-responders. However, no clinical trials have focused on identifying the type of patients who would obtain a greater benefit from receiving epinephrine. The aim of this study was to determine the variables that are strongly correlated with patient response to epinephrine in septic shock that is refractory to standard doses of norepinephrine.

\section{Materials and methods}

\section{Study setting and population}

A retrospective single-center observational study was performed using data from adult patients ( $\geq 18$ years old) who were admitted to our emergency and medical intensive care units (EMICUs) between January 2014 and December 2017. This study was approved by the Research Ethics Committee of Nagoya University, Nagoya, Japan. Nagoya University Hospital is a quaternary academic medical center with 1035 beds, including 10 EMICU and 16 surgical ICU beds.

Eligible subjects were patients with norepinephrine-refractory septic shock who had received epinephrine after admission. Sepsis was defined according to the sepsis-3 definition [13]. Septic shock was defined as a suspected infection with shock status despite volume resuscitation and vasopressor administration, as well as a serum lactate level $>2 \mathrm{mmol} / \mathrm{L}$ after $\mathrm{ICU}$ admission. We defined norepinephrine-refractoriness as persistent hypotension despite the administration of approximately $0.2 \mu \mathrm{g} / \mathrm{kg} / \mathrm{min}$ of norepinephrine. Persistent hypotension was defined as MAP less than $65 \mathrm{mmHg}$ at the time of epinephrine initiation. Patients experiencing sepsis-associated cardiac arrest and those who required mechanical circulatory support (e.g., intra-aortic balloon pumping, venous-arterial extracorporeal membrane oxygenation [VA-ECMO], or a left ventricular assisted device) prior to epinephrine administration were excluded, as were patients with do-not-resuscitate orders before ICU admission.

\section{Variables of interest and outcome measurement}

All data were retrospectively collected from electronic medical charts, including general characteristics (age, sex, and body mass index) and clinical information such as the suspected focus of infection, hemodynamic parameters, and laboratory data. The sequential organ failure assessment (SOFA) score and acute physiology and chronic health evaluation (APACHE) II score was calculated using the worst values obtained within $24 \mathrm{~h}$ after admission. At the time of epinephrine initiation, the following variables were collected; heart rate, systolic blood pressure, MAP, diastolic blood pressure, pulse pressure, central venous pressure, dose of norepinephrine, arterial blood gas data (lactate and $\mathrm{pH}$ ), and the $\mathrm{PaO}_{2}-\mathrm{FiO}_{2}$ ratio. The following variables were also recorded: time from ICU admission to epinephrine administration (time-epi), adjunctive treatment (corticosteroid use, continuous renal replacement therapy [CRRT], vasopressin administration, and the use of mechanical circulatory support after epinephrine administration), fluid volume administrated during the initial $3 \mathrm{~h}$ of epinephrine initiation, mean initial 3-h dose of epinephrine, and MAP changes $3 \mathrm{~h}$ after epinephrine initiation $(\triangle \mathrm{MAP})$. The primary endpoint was a hemodynamic improvement after epinephrine administration, as described below. The secondary endpoints were survival from shock after epinephrine administration as described below and 28-day survival.

\section{Epinephrine administration and co-intervention for septic shock patients}

Epinephrine administration was decided by the attending physician in the EMICU according to the current Japanese guideline for the management of septic shock [6]. Briefly, patients with septic shock who were hypotensive despite fluid resuscitation and the administration of $0.2 \mu \mathrm{g} / \mathrm{kg} / \mathrm{min}$ or higher dose of norepinephrine, and when other attempts failed to improve blood pressure. Vasopressin at a dose of $0.02-0.03$ units/min was administered when appropriate at least $2 \mathrm{~h}$ prior to epinephrine initiation. Low-dose corticosteroids $(200 \mathrm{mg} /$ day hydrocortisone or $40 \mathrm{mg} /$ day methylprednisolone) were also considered if appropriate prior to epinephrine use. When shock was medically uncontrollable and cardiac arrest was impending, VA-ECMO was considered in some cases.

\section{Definition of hemodynamic improvement and survival from shock after epinephrine administration}

Patients were deemed epinephrine responders if they achieved a MAP increase of $10 \mathrm{mmHg}$ or their lactate level decreased $3 \mathrm{~h}$ after epinephrine initiation; the 3-h time point was based on a prior study [14]. When there were no 3-h lactate level data available in the electronic 
chart, patients were evaluated only based on $\triangle$ MAP. Survival from shock was defined as 7-day survival after epinephrine initiation without mechanical circulatory support. The duration of 7 days was chosen since previous studies had reported that median time of death due to refractory shock was 3 days $[2,15]$.

\section{Statistical analysis}

Continuous data are summarized as mean \pm standard deviations or median and interquartile range (25th-75th percentiles), as appropriate, depending on their distribution. Categorical variables are summarized as $n(\%)$. Univariate comparison of continuous variables between responders and non-responders were analyzed using the Mann-Whitney $U$ test, and categorical variables were assessed using Fisher's exact test. As for the main endpoint analysis, multivariate logistic analysis was performed to identify independent predictors of hemodynamic improvement. Clinically significant variables, namely, SOFA score $\geq 14$, age, serum lactate level, log-transformed value of time-epi (log time-epi), and the use of steroids, were candidate factors; those significant on univariate analysis were subjected to a multivariate logistic regression model. We determined the logarithmic transformation of time-epi to fit the linear regression model based on its distribution (Additional file 1: Figure S1). We also converted the SOFA score into categorical variables; 14 was used as the cutoff based on a previous study [3]. For secondary endpoint analysis, the association between the variables and 7-day survival was assessed using a multivariate logistic regression model. To evaluate the robustness of the time-epi findings, we performed logistic regression analysis with inverse probability of treatment weighting (IPTW) using the propensity score [16] adjusted for severity (SOFA score), age, lactate, and steroid use. Odds ratios (OR) together with their 95\% confidence intervals (CIs) were estimated in these models. All statistical tests were 2 -sided, and $P<0.05$ was considered statistically significant. All statistical analyses were performed with the $R$ software (version 3.4.3) and EZR software (version 1.36) (Saitama Medical Center, Jichi Medical University, Saitama, Japan) [17].

\section{Results}

\section{Baseline characteristics}

Of the 3387 patients admitted to the EMICU, 47 had experienced norepinephrine-refractory septic shock who had received epinephrine after admission. Of this number, 41 patients were included in this study, after the exclusion of 6 patients in whom epinephrine was administered after cardiac arrest. We divided the 41 patients into those who responded to the administration of epinephrine (responders; $n=24$ ) and those who did not (non-responders; $n=17$ ) (Fig. 1). The baseline characteristics of all 41 patients are summarized in Table 1. The average overall age of patients was $63.9 \pm 15.2$ years, and the median SOFA score within $24 \mathrm{~h}$ of ICU admission was 15 (12-16). The median norepinephrine dosage at the time of epinephrine infusion was $0.2(0.17-0.25) \mu \mathrm{g} /$ $\mathrm{kg} / \mathrm{min}$, and the median time-epi was $24(12-72) \mathrm{h}$. The median fluid volume infused in the initial $3 \mathrm{~h}$ after epinephrine administration was 559 (426-808) ml of crystalloid. One patient in the non-responders group also received $250 \mathrm{ml}$ of $5 \%$ albumin solution during the 3-h evaluation. Thirty-five patients $(85 \%)$ received corticosteroid, while 20 (49\%) received vasopressin, and 28 (68\%) received CRRT prior to epinephrine administration. VA-ECMO by means of mechanical circulatory

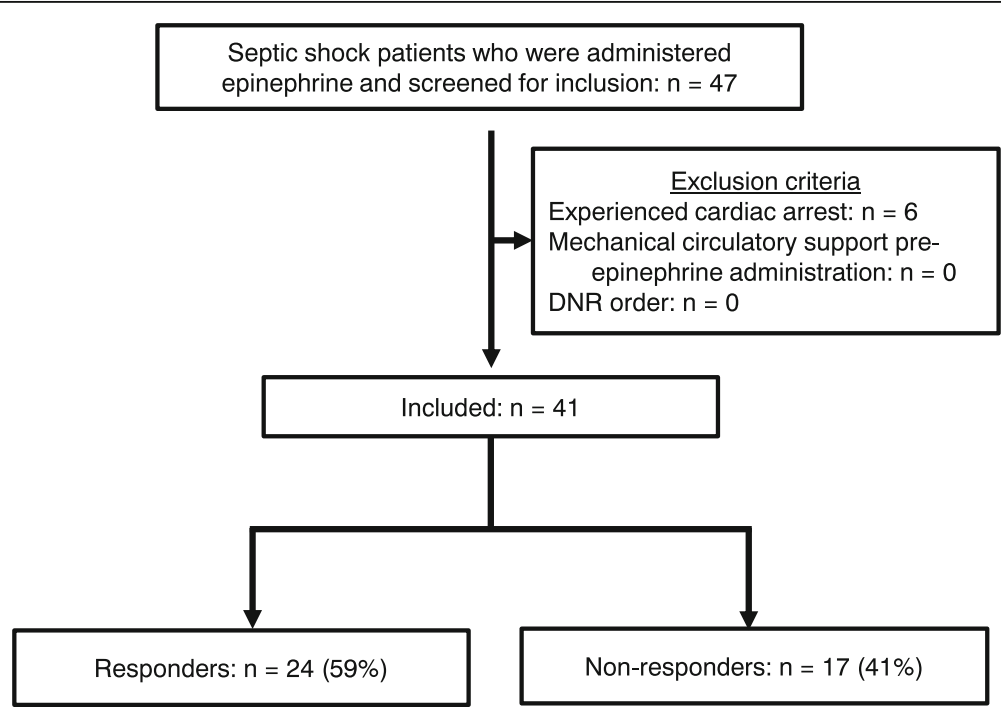

Fig. 1 Flowchart of patient selection in this study. DNR, do-not-resuscitate 
Table 1 Patient characteristics

\begin{tabular}{|c|c|c|c|c|}
\hline Characteristics & Total (41) & Responders (24) & Non-responders (17) & $P$ value \\
\hline \multicolumn{5}{|l|}{ At the day of ICU admission } \\
\hline Age, years & $63.9 \pm 15.2$ & $65.3 \pm 13.3$ & $61.8 \pm 17.6$ & 0.476 \\
\hline Male, $n(\%)$ & $25(60)$ & $15(63)$ & $10(59)$ & $>0.999$ \\
\hline BMI & $21.8 \pm 5.8$ & $22.1 \pm 7.0$ & $21.3 \pm 3.8$ & 0.657 \\
\hline SOFA score & $15(12-16)$ & $13(12-15)$ & $16(14-19)$ & 0.009 \\
\hline APACHE II score & $33(29-39)$ & $36(30-39)$ & $32(27-37)$ & 0.597 \\
\hline Creatinine, mg/dL & $1.46(0.93-2.29)$ & $1.82(0.92-2.39)$ & $1.20(0.99-1.71)$ & 0.327 \\
\hline Bilirubin, mg/dL & $1.4(0.9-4.4)$ & $1.05(0.8-1.6)$ & $1.7(1.1-7.3)$ & 0.065 \\
\hline Platelet count & $71(44-119)$ & $105(54-176)$ & $50(25-71)$ & 0.022 \\
\hline $\mathrm{CRP}, \mathrm{mg} / \mathrm{dL}$ & $10.5(6.2-18.5)$ & $9.83(5.9-15.3)$ & $10.5(6.4-22.9)$ & 0.666 \\
\hline $\mathrm{PCT}, \mathrm{ng} / \mathrm{mL}$ & $9.4(1.4-51.9)$ & $17.1(1.8-52.9)$ & $5.70(1.2-27.2)$ & 0.39 \\
\hline $\mathrm{WBC} \times 10^{3} / \mu \mathrm{L}$ & $10.7(3.7-17.1)$ & $14.0(5.1-19.5)$ & $7.4(1.2-13.1)$ & 0.153 \\
\hline $\mathrm{Hct}$ & $29.8(28.2-32.2)$ & $30.8(29.1-32.5)$ & $28.6(27.5-29.9)$ & 0.09 \\
\hline PT-INR & $1.38(1.16-1.7)$ & $1.39(1.15-1.74)$ & $1.29(1.23-1.58)$ & 0.624 \\
\hline $\mathrm{FDP}, \mu \mathrm{g} / \mathrm{ml}$ & $16.4(11.1-38.3)$ & $14.5(9.8-32.1)$ & $22.6(3.0-50.6)$ & 0.321 \\
\hline BNP, pg/ml & $432(119-1168)$ & $463(128-1169)$ & $248(119-961)$ & 0.767 \\
\hline AT3 activity, \% & $54(45-66)$ & $49(39-63)$ & $59(50-75)$ & 0.068 \\
\hline \multicolumn{5}{|l|}{ Focus of infection } \\
\hline Soft tissue, $n$ & 5 & 5 & 0 & 0.065 \\
\hline Respiratory, $n$ & 10 & 7 & 3 & 0.48 \\
\hline Central nervous system, $n$ & 2 & 1 & 1 & $>0.999$ \\
\hline Gastrointestinal, $n$ & 3 & 1 & 2 & $>0.999$ \\
\hline Hepatobiliary, $n$ & 5 & 2 & 3 & 0.633 \\
\hline Bacteremia or other, $n$ & 16 & 9 & 7 & $>0.999$ \\
\hline \multicolumn{5}{|c|}{ At the time of epinephrine infusion } \\
\hline MAP, $\mathrm{mmHg}$ & $51.3 \pm 11.2$ & $52.1 \pm 10$ & $50.3 \pm 13$ & 0.613 \\
\hline $\mathrm{PP}, \mathrm{mmHg}$ & $24.7 \pm 12$ & $26.3 \pm 11$ & $22.5 \pm 13$ & 0.317 \\
\hline $\mathrm{HR}, \mathrm{bpm}$ & $107 \pm 21$ & $103 \pm 21$ & $113 \pm 20$ & 0.149 \\
\hline $\mathrm{CVP}, \mathrm{mm} \mathrm{Hg}$ & $15(12-17)$ & $13(11-17)$ & $15(13-18)$ & 0.2 \\
\hline Lactate, mmol/L & $4.4(2.6-8.9)$ & $3.4(2.1-7.6)$ & $6.0(3.6-9.6)$ & 0.098 \\
\hline $\mathrm{pH}$ & $7.309(7.386-7.181)$ & $7.336(7.212-7.386)$ & $7.261(7.098-7.386)$ & 0.266 \\
\hline P-F ratio & $163(92-302)$ & $222(111-354)$ & $131(83-164)$ & 0.093 \\
\hline Norepinephrine, $\mu \mathrm{g} / \mathrm{kg} / \mathrm{min}$ & $0.2(0.17-0.25)$ & $0.19(0.17-0.23)$ & $0.25(0.16-0.28)$ & 0.467 \\
\hline Epinephrine, $\mu \mathrm{g} / \mathrm{kg} / \mathrm{min}^{*}$ & $0.07(0.03-0.1)$ & $0.05(0.03-0.1)$ & $0.08(0.07-0.14)$ & 0.068 \\
\hline Time-epi, $\mathrm{h}^{* *}$ & $24(12-72)$ & $18(12-24)$ & $72(6.0-144)$ & 0.004 \\
\hline \multicolumn{5}{|l|}{ Adjunctive therapy } \\
\hline Fluid infusion, ml*** & $559(426-808)$ & $559(418-762)$ & $559(447-958)$ & 0.989 \\
\hline Corticosteroid, $n(\%)$ & $35(85)$ & $20(83)$ & $15(88)$ & $>0.999$ \\
\hline Vasopressin, i (\%) & $20(49)$ & $10(42)$ & $10(59)$ & 0.35 \\
\hline CRRT, $n(\%)$ & $28(68)$ & $15(63)$ & $13(76)$ & 0.505 \\
\hline VA-ECMO, $n(\%)$ & $3(7)$ & $0(0)$ & $3(18)$ & 0.006 \\
\hline
\end{tabular}


Table 1 Patient characteristics (Continued)

\begin{tabular}{|c|c|c|c|c|}
\hline Characteristics & Total (41) & Responders (24) & Non-responders (17) & $P$ value \\
\hline \multicolumn{5}{|l|}{ Endpoints } \\
\hline$\Delta$ MAP after initiation, $\mathrm{mmHg}{ }^{* * * *}$ & $13(0-22)$ & $20(14-29)$ & $-1(-7$ to 6$)$ & $<0.001$ \\
\hline Lactate $3 \mathrm{~h}$ after initiation, $\mathrm{mmol} / \mathrm{L}$ & $6.4(3.1-11.5)$ & $4.2(2.8-9.0)$ & $11.4(5.1-13.8)$ & 0.039 \\
\hline Survival from shock, $n(\%)^{* * * * *}$ & $25(61)$ & $22(92)$ & $3(18)$ & $<0.001$ \\
\hline 28-day survival, $n(\%)$ & $23(56)$ & $20(83)$ & $3(18)$ & $<0.001$ \\
\hline
\end{tabular}

ICU intensive care unit, $B M I$ body mass index, SOFA Sequential Organ Failure Assessment, APACHE acute physiology and chronic health evaluation, $C R P$ C-reactive protein, $P C T$ procalcitonin, WBC white blood cell, $P T$-INR: prothrombin time-international normalized ratio, $F D P$ : fibrin degradation product, $B N P$ brain natriuretic peptide, $A T 3$ anti-thrombin 3, MAP mean arterial pressure, $P P$ pulse pressure, $H R$ heart rate, CVP central venous pressure, CRRT continuous renal replacement therapy, VA-ECMO veno-arterial membrane oxygenation * Median epinephrine dose of initial 3-h administration

**Time of epinephrine administration after ICU admission

***Fluid administration during initial $3 \mathrm{~h}$ of epinephrine use

****MAP change $3 \mathrm{~h}$ after epinephrine initiation

*****7-day survival without the use of mechanical circulatory support

Data are presented as the mean \pm standard deviation, the median and interquartile ranges $(25-75 \%$ percentile), or as absolute frequencies with percentages

support for septic cardiomyopathy was introduced in 3 patients. Responders had a higher rate of survival from shock ( $92 \%$ vs. $18 \%, P<0.001)$, and 28 -day survival $(83 \%$ vs. $18 \%, P<0.001)$.

\section{Factors associated with responsiveness to epinephrine administration}

We performed univariate analysis to identify factors that were strongly associated with responsiveness to the administration of epinephrine (Table 2). The SOFA score within $24 \mathrm{~h}$ of ICU admission and the log time-epi were significantly associated with responsiveness to epinephrine. We subjected these 2 variables to multivariate logistic regression analysis, which showed them to be independently associated with responsiveness to epinephrine (OR 0.48, 95\% CI 0.27-0.87, $P=0.011$ and OR $0.19,95 \%$ CI $0.04-0.88, P=0.034$, respectively) (Table 3 ). To evaluate the robustness of time-epi as a factor, we also investigated the OR of epinephrine response upon its later administration using the IPTW-propensity score, which was 0.07 (95\% CI $0.02-0.21 ; P=0.001)$ (Table 3). This suggested that epinephrine administration within $24 \mathrm{~h}$ of ICU admission was significantly associated with a better response. Furthermore, we found that the $\log$ time-epi was significantly associated with

Table 2 Univariate analyses of factors predicting epinephrine response

\begin{tabular}{llll}
\hline Variables & OR & $95 \% \mathrm{Cl}$ & $P$ value \\
\hline SOFA score $\geq 14$ & 0.21 & $0.05-0.8$ & 0.022 \\
Age & 1.02 & $0.97-1.06$ & 0.467 \\
Lactate & 0.91 & $0.8-1.05$ & 0.19 \\
Log time-epi & 0.21 & $0.06-0.73$ & 0.013 \\
Steroid & 0.67 & $0.11-4.13$ & 0.663 \\
\hline
\end{tabular}

OR odds ratio, $\mathrm{Cl}$ confidence interval, SOFA Sequential Organ Failure Assessment, time-epi time of epinephrine administration after intensive care unit admission survival from shock and 28-day survival, whereas the SOFA score was not (Table 4).

\section{Discussion}

A major reason for the efficacy of epinephrine in treating refractory septic shock not being clearly established is that previous studies included the entire septic shock patient population at a given facility, despite the fact that some patients respond more favorably to epinephrine than others. In the present study, we retrospectively analyzed the data of 41 septic patients who were refractory to norepinephrine and found that the timing of epinephrine administration, as well as the SOFA score within the first $24 \mathrm{~h}$ of ICU admission, was strongly associated with responsiveness to epinephrine.

Relationship between the response to epinephrine, the timing of its administration, and the SOFA score

Our finding that late-stage epinephrine administration was associated with low response rate may be explained by the fact that catecholamine resistance in patients with sepsis changes over time. This change is caused by intracellular calcium overload and impairment of intracellular signaling via adrenergic beta receptors in cardiomyocytes

Table 3 Multivariable logistic regression analyses of predicting factors of epinephrine response

\begin{tabular}{|c|c|c|}
\hline Variable & $\mathrm{OR}(95 \% \mathrm{Cl})$ & $P$ value \\
\hline Log time-epi & $0.48(0.27-0.84)$ & 0.011 \\
\hline SOFA score $\geq 14$ & $0.19(0.04-0.88)$ & 0.034 \\
\hline \multicolumn{3}{|c|}{ Adjusted using IPTW-propensity score* } \\
\hline Time-epi $\geq 24 \mathrm{~h}$ & $0.07(0.02-0.21)$ & $<0.001$ \\
\hline
\end{tabular}

OR odds ratio, $\mathrm{Cl}$ confidence interval, Time-epi time of epinephrine administration after intensive care unit admission, SOFA Sequential Organ Failure Assessment, IPTW inverse probability of treatment weighting

*Variables used to calculate the propensity score included age, lactate, steroid use, and SOFA score 
Table 4 Multivariable logistic analyses of predicting factors for survival from shock and 28-day survival

\begin{tabular}{lll}
\hline Variable & OR $(95 \% \mathrm{Cl})$ & $P$ value \\
\hline Association with survival from shock* & \\
Log time-epi & $0.42(0.23-0.77)$ & 0.005 \\
SOFA score $\geq 14$ & $0.41(0.09-1.84)$ & 0.247 \\
Association with 28-day survival & & \\
Log time-epi & $0.14(0.04-0.57)$ & 0.006 \\
SOFA score $\geq 14$ & $0.6(0.14-2.53)$ & 0.488 \\
\hline
\end{tabular}

OR odds ratio, $\mathrm{Cl}$ confidence interval, Time-epi time of epinephrine administration after intensive care unit admission, SOFA Sequential Organ Failure Assessment

*7-day survival without the use of mechanical circulatory support

$[18,19]$. If epinephrine is administered in the later stages of septic shock, the increased catecholamine resistance may reach a level that prevents any response to epinephrine. To the best of our knowledge, ours is the first study to show that the time course of septic shock is associated with the hemodynamic response to catecholamines. Furthermore, our study confirmed a previous finding by Conrad et al. that a higher SOFA score is strongly associated with catecholamine hypo-responsiveness [3].

Interestingly, IPTW analysis revealed that the administration of epinephrine within $24 \mathrm{~h}$ improved responsiveness compared to later administration. A randomized control trial failed to show any efficacy of epinephrine administration in treating septic shock, which might be because in that study the mean time for patient randomization after ICU admission was $48 \mathrm{~h}$ [12], which appears to be too long to observe a benefit for epinephrine administration and more likely to observe a lack of response to epinephrine administration. Our findings may provide insight into the reason negative data were produced in the abovementioned study.

\section{The analysis of secondary outcome}

While 28-day survival is commonly used in the field of critical care, we also chose 7-day survival after epinephrine initiation without mechanical circulatory support as the secondary outcome to focus on cause-specific mortality of refractory shock and to make sure of consistency in the findings between primary and secondary endpoint analyses. The reason for death in sepsis is multifactorial [2]. Several studies have reported that the median time to death in refractory shock was 3 days [2, 15]. Therefore, we consider that 7 days were long enough to detect death due to shock. VA-ECMO might be an effective rescue therapy for refractory shock in some septic shock patients [20]. In our study group, 3 patients who received VA-ECMO therapy were considered hemodynamically unstable after using epinephrine. We decided that they would die of shock death if ECMO was not used. Therefore, we classified those who received ECMO as non-survivors even if they survived longer than 7 days. By using this outcome, we showed that the timing of epinephrine administration was linked to some extent with the short-term efficacy of epinephrine in preventing death from septic shock. Also, we found the results of analyses were similar between secondary endpoints.

While late administration of epinephrine ( $>24 \mathrm{~h}$ ) was associated with worse short-term outcome, initial SOFA score was not. We need a larger sample size to determine whether initial SOFA score is useful in predicting the short-term patient outcome after epinephrine use. In any case, late stages of septic shock rarely benefit from epinephrine administration and should be excluded from future clinical trials.

\section{Effects of co-interventions over the results}

One of the major limitations of this study was that confounding bias of co-interventions (fluid, corticosteroids, vasopressin) cannot be excluded, owing to the limited sample size and retrospective nature of the study. Although we used epinephrine when prior co-interventions failed, these interventions can be possible confounders. To minimize the effect of confounders, a definitive treatment protocol must be devised. It would be interesting to evaluate other indicators in epinephrine responders in future prospective studies with a certain protocol.

\section{Other limitations}

Our study has several other limitations. First, it was a single-center retrospective observational study, and the administration of epinephrine was decided based on the clinical preferences of attending physicians. Although selection bias cannot be completely ruled out, the baseline hemodynamic parameters (MAP, heart rate, serum lactate levels, etc.) were similar between responders and non-responders. Also, we selected arbitrary endpoints for the evaluation of variables. The clinical importance of these endpoints should be validated in the future. Second, our study involved a small-sized cohort; this may explain our inability to detect a significant association between the SOFA score and short-term outcome, in contrast to a previous report [3]. Future study with a large sample size should be performed to see the effect of variables (e.g., lactate, and other laboratory data) for the hemodynamic response. Third, we did not evaluate the relationship between the site of infection and epinephrine response; this should be evaluated in a future trial. Finally, long-term outcomes were not investigated because of the study design and sample size; it would be of great interest to determine the association between epinephrine response and such long-term outcomes. 


\section{Conclusions}

Early administration of epinephrine after ICU admission (i.e., within $24 \mathrm{~h}$ ) is associated with hemodynamic improvement in norepinephrine-refractory septic shock.

\section{Additional file}

Additional file 1: Figure S1. The time distribution of epinephrine initiation after intensive care unit admission. The minimum and maximum times were 3 and $696 \mathrm{~h}$, respectively, while the median (interquartile range) was $24(12-72) \mathrm{h}$. (PPTX $1525 \mathrm{~kb}$ )

\begin{abstract}
Abbreviations
APACHE: Acute physiology and chronic health evaluation; Cl: Confidence interval; CRRT: Continuous renal replacement therapy; EMICU: Emergency and medical intensive care unit; ICU: Intensive care unit; IPTW: Inverse probability of treatment weighting; MAP: Mean arterial pressure; OR: Odds ratio; SOFA: Sequential Organ Failure Assessment; time-epi: Time from ICU admission to epinephrine administration; VA-ECMO: Venous-arterial extracorporeal membrane oxygenation
\end{abstract}

\section{Acknowledgements}

This work was supported by the Clinical Research Program at Nagoya University. Part of this study has been presented at the EuroAsia conference of European Society of Intensive Care Medicine (Hong Kong, China, April 2018) [21]. We thank the residents, fellows, paramedical staff, and Teruko Mizutani (the administrative assistant) for data collection and treatment support in our ICU.

\section{Funding}

This research did not receive any specific grant from funding agencies in the public, commercial, or not-for-profit sectors.

\section{Availability of data and materials}

The dataset supporting the conclusions of this article is available from the corresponding author on reasonable request.

\section{Authors' contributions}

DK and MN conceived and designed the study. DK, MH, and TY gathered the data set. KN, KT, and SM analyzed the data. DK, MN, KN, and AN interpreted the data. DK, MN, KN, and NM contributed to the writing of the manuscript. All authors read and approved the final manuscript.

\section{Ethics approval and consent to participate}

The institutional review board approved this study. For this type of retrospective study, patient consent was not required.

\section{Consent for publication}

Not applicable.

\section{Competing interests}

The authors declare that they have no competing interests.

\section{Publisher's Note}

Springer Nature remains neutral with regard to jurisdictional claims in published maps and institutional affiliations.

\section{Author details}

'Department of Emergency and Critical Care, Nagoya, University Graduate School of Medicine, Tsurumai-cho 64, Syowa-ku, Nagoya, Aichi 4668560, Japan. ${ }^{2}$ Department of Biostatistics, Nagoya University Graduate School of Medicine, Tsurumai-cho 64, Syowa-ku, Nagoya, Aichi 4668560, Japan.
Received: 29 December 2018 Accepted: 26 March 2019

Published online: 05 April 2019

\section{References}

1. Avni T, Lador A, Lev S, Leibovici L, Paul M, Grossman A. Vasopressors for the treatment of septic shock: systematic review and meta-analysis. PLoS One. 2015;10:e0129305. https://doi.org/10.1371/journal.pone.0129305.

2. Moskowitz A, Omar Y, Chase M, Lokhandwala S, Patel P, Andersen LW, et al. Reasons for death in patients with sepsis and septic shock. J Crit Care. 2017; 38:284-8. https://doi.org/10.1016/j.jcrc.2016.11.036.

3. Conrad M, Perez P, Thivilier C, Levy B. Early prediction of norepinephrine dependency and refractory septic shock with multimodal approach of vascular failure. J Crit Care. 2015;30:739-43. https://doi.org/10.1016/j.jcrc. 2015.03.029.

4. Auchet T, Regnier MA, Girerd N, Levy B. Outcome of patients with septic shock and high-dose vasopressor therapy. Ann Intensive Care. 2017;7:43. https://doi.org/10.1186/s13613-017-0261-X.

5. Jenkins CR, Gomersall CD, Leung P, Joynt GM. Outcome of patients receiving high dose vasopressor therapy: a retrospective cohort study. Anaesth Intensive Care. 2009:37:286-9.

6. Nishida O, Ogura H, Egi M, Fujishima S, Hayashi Y, Iba T, et al. The Japanese clinical practice guidelines for management of sepsis and septic shock 2016 (J-SSCG 2016). J Intensive Care. 2018;6:7. https://doi.org/10.1186/s40560-0170270-8.

7. Rhodes A, Evans LE, Alhazzani W, Levy MM, Antonelli M, Ferrer R, et al. Surviving sepsis campaign: international guidelines for management of sepsis and septic shock: 2016. Intensive Care Med. 2017;43:304-77. https:// doi.org/10.1007/s00134-017-4683-6.

8. Zausig YA, Geilfus D, Missler G, Sinner B, Graf BM, Zink W. Direct cardiac effects of dobutamine, dopamine, epinephrine, and levosimendan in isolated septic rat hearts. Shock. 2010;34:269-74. https://doi.org/10.1097/ SHK.0b013e3181cd877b.

9. Le Tulzo Y, Seguin P, Gacouin A, Camus C, Suprin E, Jouannic I, et al. Effects of epinephrine on right ventricular function in patients with severe septic shock and right ventricular failure: a preliminary descriptive study. Intensive Care Med. 1997;23:664-70

10. Mahmoud KM, Ammar AS. Norepinephrine supplemented with dobutamine or epinephrine for the cardiovascular support of patients with septic shock. Indian J Crit Care Med. 2012;16:75-80. https://doi.org/10.4103/0972-5229.99110.

11. Myburgh JA, Higgins A, Jovanovska A, Lipman J, Ramakrishnan N, Santamaria J, Study investigators CAT. A comparison of epinephrine and norepinephrine in critically ill patients. Intensive Care Med. 2008;34:2226-34. https://doi.org/10.1007/s00134-008-1219-0.

12. Annane $D$, Vignon $P$, Renault A, Bollaert PE, Charpentier C, Martin C, et al. Norepinephrine plus dobutamine versus epinephrine alone for management of septic shock: a randomised trial. Lancet. 2007;370:676-84.

13. Singer M, Deutschman CS, Seymour CW, Shankar-Hari M, Annane D, Bauer $M$, et al. The third international consensus definitions for sepsis and septic shock (Sepsis-3). JAMA. 2016;315:801-10. https://doi.org/10.1016/S01406736(07)61344-0.

14. Khanna A, English SW, Wang XS, Ham K, Tumlin J, Szerlip H, et al. Angiotensin II for the treatment of vasodilatory shock. N Engl J Med. 2017; 377:419-30. https://doi.org/10.1056/NEJMoa1704154.

15. Döpp-Zemel D, Groeneveld AB. High-dose norepinephrine treatment: determinants of mortality and futility in critically ill patients. Am J Crit Care. 2013:22:22-32. https://doi.org/10.4037/ajcc2013748.

16. Austin PC. Variance estimation when using inverse probability of treatment weighting (IPTW) with survival analysis. Stat Med. 2016;(30):5642-55. https:// doi.org/10.1002/sim.7084.

17. Kanda Y. Investigation of the freely available easy-to-At the time of epinephrine infusionstatistics. Bone Marrow Transplant. 2013;48:452-8. https://doi.org/10.1038/bmt.2012.244

18. Matsuda N, Hattori Y, Akaishi Y, Suzuki Y, Kemmotsu O, Gando S. Impairment of cardiac beta-adrenoceptor cellular signaling by decreased expression of $\mathrm{G}$ (s alpha) in septic rabbits. Anesthesiology. 2000;93:1465-73.

19. Rudiger A, Singer M. Mechanisms of sepsis-induced cardiac dysfunction. Crit Care Med. 2007:35:1599-608. https://doi.org/10.1097/01.CCM.0000266683. 64081.02

20. Bréchot $N$, Luyt CE, Schmidt $M$, Leprince $P$, Trouillet JL, Léger $P$, et al. Venoarterial extracorporeal membrane oxygenation support for refractory 
cardiovascular dysfunction during severe bacterial septic shock. Crit Care Med. 2013;41:1616-26. https://doi.org/10.1097/CCM.0b013e31828a2370.

21. Kasugai D, Nishikimi M, Matsuda N. Efficacy of epinephrine for norepinephrine-refractory septic shock: European Society of Intensive Care Medicine presentation. http://mediatheque.cyim.com/mediatheque/media. aspx?mediald $=43844 \&$ channel $=23107$. [Accessed 6 Dec 2018]

Ready to submit your research? Choose BMC and benefit from:

- fast, convenient online submission

- thorough peer review by experienced researchers in your field

- rapid publication on acceptance

- support for research data, including large and complex data types

- gold Open Access which fosters wider collaboration and increased citations

- maximum visibility for your research: over $100 \mathrm{M}$ website views per year

At BMC, research is always in progress.

Learn more biomedcentral.com/submissions 